\title{
Towards Neuro-Inspired Electronic Oscillators Based on The Dynamical Relaying Mechanism
}

\author{
Gianluca Susi ${ }^{\# *}$, Simone Acciarito ${ }^{+}$, Teodoro Pascual ${ }^{*}$, Alessandro Cristini $^{+}$, Fernando Maestú $^{*}$ \\ " Department of Civil Engineering and Computer Science (DICII), University of Rome "Tor Vergata", Rome, Italy \\ E-mails: gianluca.susi@uniroma2.it
}

* Department of Experimental Psychology, Cognitive Processes and Logopedy, Complutense University of Madrid, Madrid, Spain

E-mail: gsusi@ucm.es; teodorop@ucm.es;fmaestuu@psi.ucm.es

${ }^{+}$Department of Electronic Engineering, University of Rome “Tor Vergata”, Rome, Italy

E-mail: simone.acciarito@uniroma2.it ; alessandro.cristini@uniroma2.it

\begin{abstract}
Electronic oscillators are used for the generation of both continuous and discrete signals, playing a fundamental role in today's electronics. In both contexts, these systems require stringent performances such as spectral purity, low phase noise, frequency and temperature stability. In state of the art oscillators the preservation of some of these aspects is jeopardized by specific critical issues, e.g., the sensitivity to load capacitance or the component aging over time. This leaves room for the search of new technologies for their realization. On the other hand, in the last decade electronics has been influenced by a growing number of neuro-inspired mechanisms, which allowed for alternative techniques aimed at solving some classical critical issues. In this paper we present an exploratory study for the development of electronic oscillators based on the neuro-inspired mechanism dynamical relaying, which relies on a structure composed of three delay coupled units (as neurons or even neuron populations) able to resonate and self-organise to generate and maintain a given rhythm with great reliability over a considerable parameter range, showing robustness to noise. We used the recent leaky integrated and fire with latency (LIFL) as neuron model. We have initially developed the mathematical model of the neuro-inspired oscillator, and implemented it using Matlab®; then, we have realized the schematic of such system in PSpice ${ }^{\circledR}$. Finally, the model has been validated to verify whether it observes the fundamental properties of the dynamical relaying mechanisms described in computational neuroscience studies, and if the circuit implementation presents the same behaviour of the mathematical model. Validation results suggest that the dynamical relaying mechanism can be proficuously taken in consideration as alternative strategy for the design of electronic oscillators.
\end{abstract}

Keywords - LIFL neuron model; electronic oscillators; dynamical relaying; spiking neural networks.

\section{INTRODUCTION}

Electronic oscillators are widely used in many electronic devices, for the generation of both sinusoidal and discrete signals [1]. The preservation of some of their characteristics over time, as spectral purity, low phase noise, frequency and temperature stability, is threatened by specific critical issues, e.g., the component aging [2-5]. In addition, the presence of several cores in modern central processing units (CPUs) brings out as new problem the synchronization between them [6]. This leaves room for the search of new technologies for the realization of electronics oscillators, and the exploration of new synchronization strategies. On the other hand, during the last decade electronics has been influenced by a growing number of biologically-inspired mechanisms, which allowed for alternative techniques aimed at solving critical issues (e.g., [7],[8]).

The dynamical relaying mechanism [9]-[11] is gaining a growing interest in the field of computational neuroscience. It relies on a specific network motif based on three delay coupled nodes, e.g., neurons or neuronal groups, and allows to generate oscillations with constant frequency and high robustness with respect to parameter mismatch and system noise [12]. Such network motif is ubiquitous in different areas, like brain-, social-, or information centric-networks [9],[12]-[15]. Previous studies regarding neural computation show that a pervasive dynamical regime is established in structures of this type, where outer elements of the chain are phase locked at 0-lag, and the middle one acts as a dynamical relay element between the other two [10],[16]. 


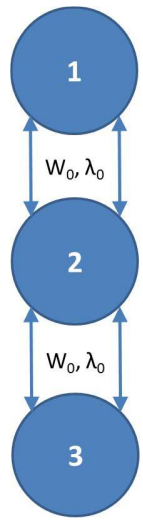

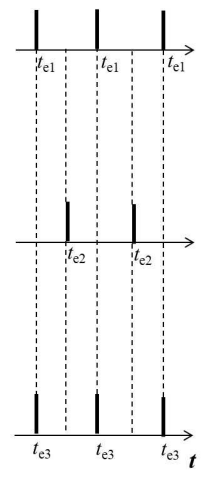

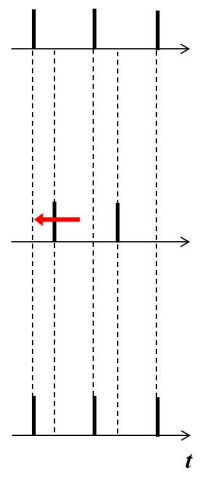

b)

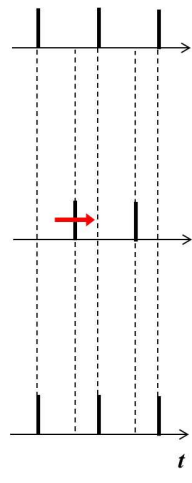

c)
Fig.1: Basic structure for the dynamical relying mechanism, composed of three delay coupled oscillating units. Depending on the delay time and coupling strength $\left(\mathrm{w}_{0}, \lambda_{0}\right)$, three synchrony modes appear prominently: a) driven synchrony (where the central pulse is in antiphase with the one of the outer oscillators), b) slave synchrony (where the central pulse is anticipated), c) pacemaker synchrony (where the central pulse is postponed); the higher SQ has been reported in the driven synchrony regime (see [17]).

Depending on the coupling strength and the delay among the nodes, different synchronization regimes can appear with such configuration. Among the various authors that have studied this mechanism, Viryiopase et al. [17] performed extensive simulations using phase oscillators and spiking neurons as nodes, arriving to the identification of three main different synchronization regimes: driven synchrony, slave synchrony and pacemaker synchrony, depending on the position of the signal produced by the relay element compared to the external ones (i.e., central, left, and right respectively, see fig. 1 ).

The authors evaluate the precision of the structure through the synchronization quality (SQ) index, that reflects the fraction of initial random phase combinations of the three nodes that lead to stable and synchronous firing of the outer ones $\left(\left|\Delta t_{\mathrm{e} 1} t_{\mathrm{e} 3}\right|<0.02\right.$ times the oscillation period).

This metric is of particular importance because, since both of the outer nodes are afferent to the central one, in such structural symmetry their synchronization is an index of stability of the overall structure. Among the three regimes, the driven synchrony regime has been shown to be an asymptotically stable mode, characterized by a SQ up to $100 \%$, for a very large range of system parameters [17], which makes us consider the driven synchrony the most interesting regime for this work because it opens new scenarios in terms of fault tolerance and robustness to noise.

To reproduce this phenomenon, we have built the neuroinspired system in form of a spiking neural network (SNN). The SNN that we implemented is based on the biologically plausible leaky integrate and fire with latency (LIFL) neuron model [18]-[22]. Firstly, we implemented the system equations in Matlabß; then, we have realized the schematic of such system in PSpice ${ }^{\circledR}$, exploiting a circuit previously developed by our group [23],[24].

Finally, the model has been validated to verify whether 1) it observes the fundamental properties of the dynamical relaying mechanisms described in computational neuroscience studies, and 2) if the circuit implementation presents the same behaviour of the mathematical model.

\section{MATERIAL AND METHOD}

A. Large-scale synchronization phenomena and organization of brain rhythms in the brain seem to be supported by the dynamical relaying mechanism

In the 1920s Hans Berger performed the first electroencephalogram in history and discovered that the brain was a generator of oscillations: synchronized rhythmic patterns of electrical activity produced by neurons, spinal cord, and autonomic nervous system. Brain oscillations have been traditionally categorized into five bands of frequency: $\delta$ (0.5-3.5 Hz), $\theta(3.5-7.5 \mathrm{~Hz}), \alpha(7.5-12 \mathrm{~Hz}), \beta(12-30 \mathrm{~Hz})$ and $\gamma(>30 \mathrm{~Hz})$ [25]-[27]. Neuronal rhythms arise from the interaction of multiple irregularly firing neurons within a brain structure or between different brain structures, and reflect competition between excitation and inhibition processes. Therefore, they can be generated by different mechanism in different structures, and the contribution of a specific frequency relays on the function of the brain system that supports in [25].

The dynamical relaying has gathered great interest as a candidate mechanism for long-range zero-lag synchronization mechanism between different brain areas, mediated by a third (relay) node (e.g. cortico-thalamocortical circuits), robust to a broad range of conduction delays and cell types [10]. In addition, recent literature in the field of neuroscience depicts it as the responsible of the cross-frequency-coupling in the brain [11], i.e., the coordination among different rhythms.

Synchronization phenomena among electronic circuits whose dynamics is relayed by a third parameter-matched circuit have been already discussed in literature (see [16]). The allaged importance of the dynamical relaying mechanism suggests its use for the realization of neuroinspired devices.

\section{B. Mathematical models of the dynamic relaying oscillator realized in this study and its implementation in Matlab}

LIFL neuron model is similar to the classical leaky integrate and fire, but it contemplates the presence of an important neurocomputational feature, called spike latency (see fig.2), which characterizes the neuron behavior in the suprathreshold region: when its internal state $S$ (i.e., the membrane potential) reaches the spiking threshold $S_{\mathrm{th}}$, the firing is not instantaneous, but it occurs after a statedependent continuous time delay $t_{\mathrm{f}}$ (i.e., the spike latency):

$$
t_{\mathrm{f}}=1 /(S-1)
$$

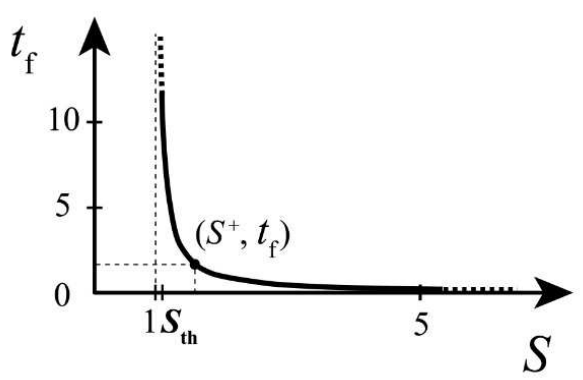

Fig.2: Spike latency curve, i.e., graphical representation of equation (1) (see [18]). 
Then, the LIFL neuron can be characterized by a voltagelike state variable $f[0, \infty]$. When exposed to a DC input, it behaves as pulse generator, which its cycle (LIFL cycle, $L C$ ) is characterized by three different phases: its internal state increases monotonically, passing for the "passive mode" $\left(t_{\text {pas }}\right)$ and then for the "active mode" $\left(t_{\text {act }}\right)$ phases, where it terminates with the generation of a spike (see fig.3). The state variable is subsequently reset to zero, where it stands for an interval known as absolute refractory period $\left(t_{\text {arp }}\right)$, and then the cycle repeats.

$$
\begin{array}{cl}
S=S_{a}+P_{\mathrm{r}} P_{\mathrm{w}}-L d \Delta t & \text { for } S<S_{\mathrm{th}} \\
S=S_{\mathrm{a}}+P_{\mathrm{r}} P_{\mathrm{w}}+\left[\left(S_{\mathrm{a}}-1\right)^{2} \Delta t /\left(S_{\mathrm{a}}-1\right)^{2} \Delta t\right] & , \text { for } S \geq S_{\mathrm{th}}
\end{array}
$$

In equations (2)-(3), $S_{\mathrm{a}}$ represents the previous state, $L d$ the subthreshold linear decay, and $\Delta t$ is the temporal difference between two consecutive incoming spikes. When $S \geq S_{\text {th }}$ (suprathreshold region), the neuron becomes active and it is ready to produce a spike, remaining still sensitive to incoming spikes. Thus, as time advances and new spikes arrive, the state $S$ is properly increased, and $t_{\mathrm{f}}$ is evaluated. In this region, for each new state the bijective relationship (1) is evaluated, leading to equation (3) [22].

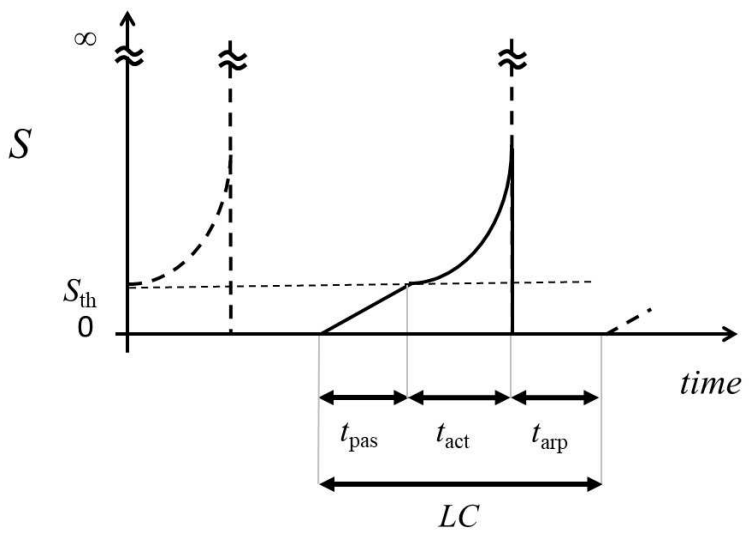

Fig.3: Cicle of the LIFL neuron when excited by a DC current of sufficient value. The internal state can be expressed as function of the phase variable such that $\varphi[0, \mathrm{LC}] \rightarrow f[0, \infty]$.

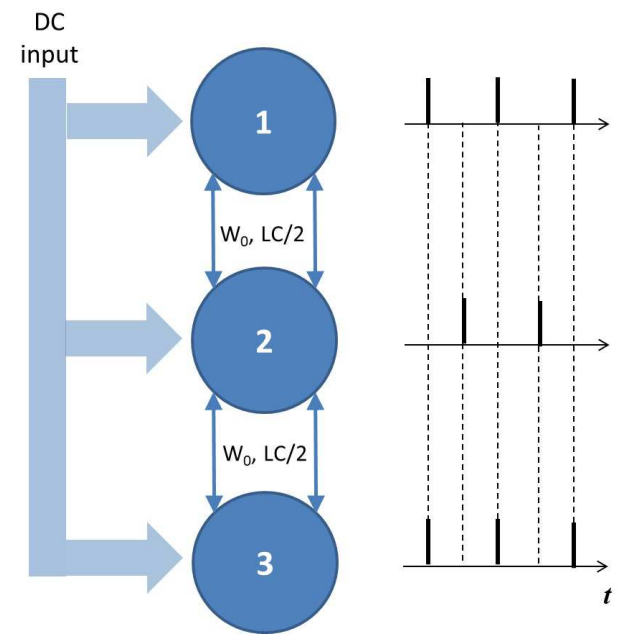

Fig.4: Schematic of the overall network model, in the driven synchrony regime

In order to synthesize the overall network, we have taken in account the ideal conditions to obtain driven synchrony regime, i.e., that the period of all oscillators is twice the conduction delay (see [17]). We have tuned the structure as follows: 1) we adjusted the DC input to obtain the desired period for $L C ; 2$ ) we set $W_{0}$ sufficiently high to achieve the entrainment among the groups, but at the same time small enough not to generate a significant variation of the period $L C$; 3) we set $\lambda_{0}=L C / 2$ to achieve the desired driven synchrony regime (see fig.4). We implemented the structure in Matlab®, using the LIFL equations described in [22].

In the model we have adimensional quantities. We finally arrived, as ideal values for the regime of interest, to $L C=10$, $\lambda_{0}=5$ and $W_{0}=0.15$.

We have executed a battery of 900 simulations in the neighborhood of the parameters $\lambda_{0}$ and $W_{0}$ and analyzed the results. All the results obtained are displayed in graphical form.

\section{Framework for PSpice simulations and validation of the circuit properties}

In this phase, we have generated the dynamical relay model with Orcad PSpice, consisting in three LIFL neurons connected with delay, using the implementation of the LIFL neuron presented in [23]. The neuron is composed of 8 logical subsystems (fig.5):

1. Integrator circuit (I): composed of the input stage of an OTA;

2. Internal state (IS): RC group;

3. Minimum Threshold (mT): input stage of a differential amplifier;

4. Non Linear Element (NLE): common-source amplifiers, peak detectors (diode and capacitor), shunt, and voltage translators;

5. Latency Generation (LG): ramp generator, a monostable and an adder;

6. Maximum Threshold (MT): input stage of a differential amplifier;

7. Pulse Generator (PG): monostable and an output buffer;

8. Refractory $(\mathrm{R})$ : peak detector with loss (diode - capacitor - resistor) and a buffer.

We have sized the circuit in order to obtain a period of 40 $\mu \mathrm{s}$, in the driven synchrony regime. From this system we have obtained the netlist with the signals that we want to monitor (i.e., coupling delay and weight). Once the netlist of the system saved in a text file is obtained, we proceeded to modify it with a Matlab script in the following way: the netlist file generated first read by Matlab, the value of the variables we want to update are written in order to obtain a new netlist with the values to simulate (yellow block of fig.6). Subsequently, PSpice is called from Matlab with the new modified netlist as argument (green block). After the simulation, we process the results (dark blue). The simulations continue until all the possible combinations of the variables we want to simulate have been analyzed (cyan block). Finally, after the last step, all the results obtained are displayed in graphical form (see fig.6).

For the simulated circuit we arrived to the following set of ideal values for the regime of interest, $L C=40 \mathrm{us}, \lambda_{0}=20 \mu \mathrm{s}$ and $W_{0}=0.15 \%$ of the $S_{\text {th }}$. For both Matlab and PSpice sets of simulations, SQ has been evaluated over 10 trials for each combination of delay and weight (see fig.7). 


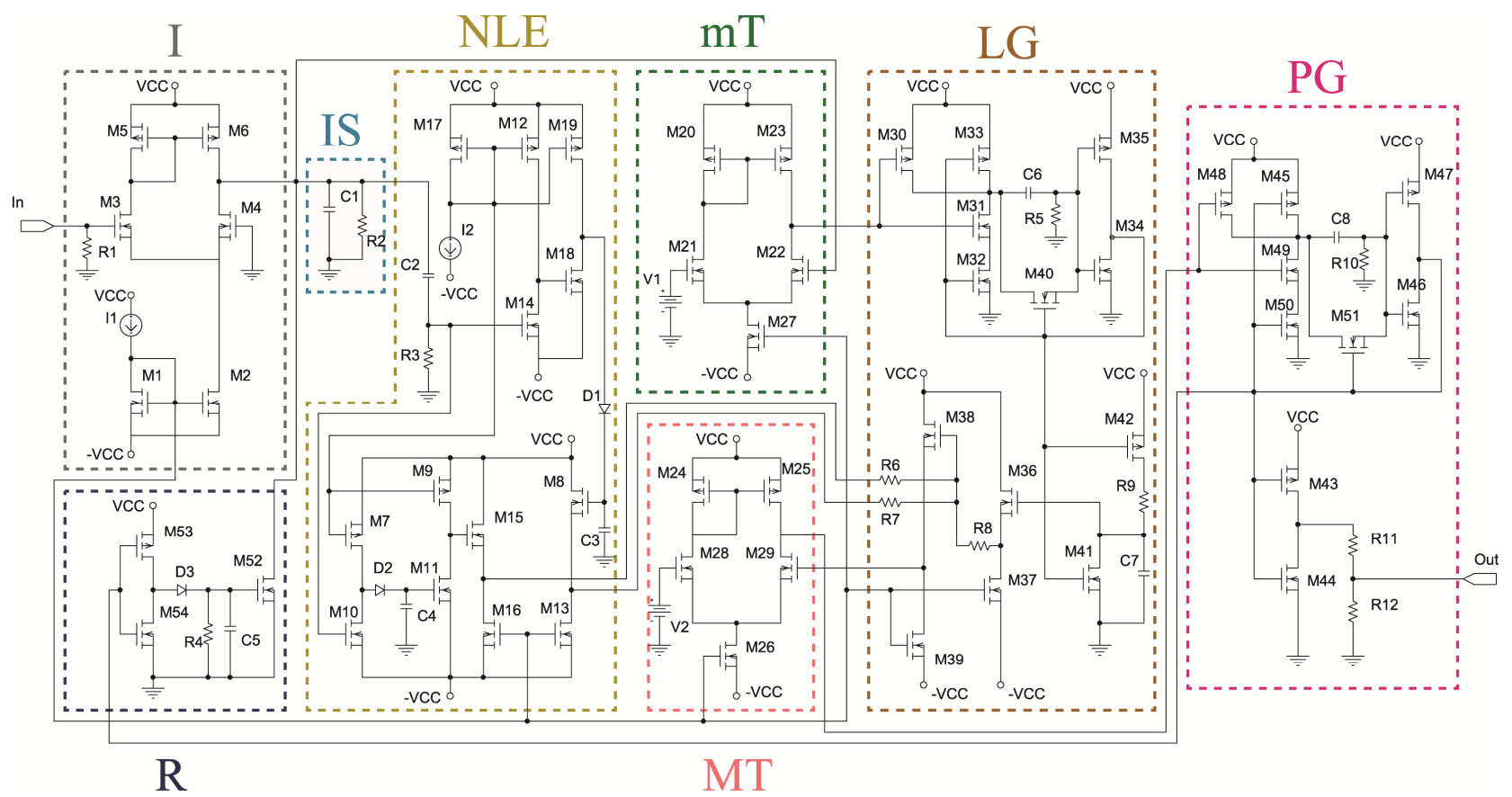

Fig.5: Scheme of the neuron circuit. I: integrator circuit; IS: internal state block; mT: minimum threshold block; NLE: non-linear element block; LG: latency generation block; MT: maximum threshold block; PG: pulse generator block; R: refractory block (modified from [24])

\section{RESULTS AND DISCUSSION}

Simulation results confirm that the LIFL-based model, when in driven synchrony regime, preserve reliability for a quite large range of the parameters, as described in [17], and that the circuit implementation accurately emulates the behaviour of the mathematical model.

This suggests that the dynamical relaying mechanism can be proficiently taken in consideration as alternative strategy for the design of electronic oscillators. Interestingly, Viriyopase et al. [17] have shown that in these networks the stability is facilitated when spike timing dependent plasticity (STDP) is implemented in the synapses, which is also capable to convert slave synchrony to driven synchrony, and thus to expand the possibility of reaching such a stable regime. This suggests for future works the realization of the dynamical relaying motif with plastic synapses [28].

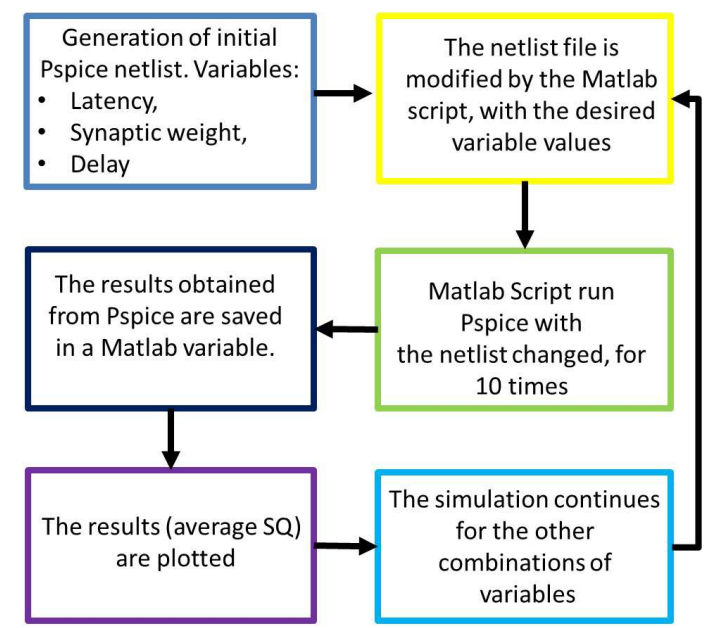

Fig.6: Pipeline of the implemented method for the validation of the circuit
Neural networks are being used today for a plenty of application, ranging from classification [29],[30], prediction [31]-[34], or optimization [35] problems, as well as for the emulation of brain dynamics [36],[37]. Considering the latter context, the dynamical relaying mechanism has been reproduced with larger neural networks, where single neurons are replaced by neuron populations, and links are replaced by bundles of connections [10],[12]. These aspects open an interesting scenario for the hardware redundancy and then for the development of fault tolerant devices.

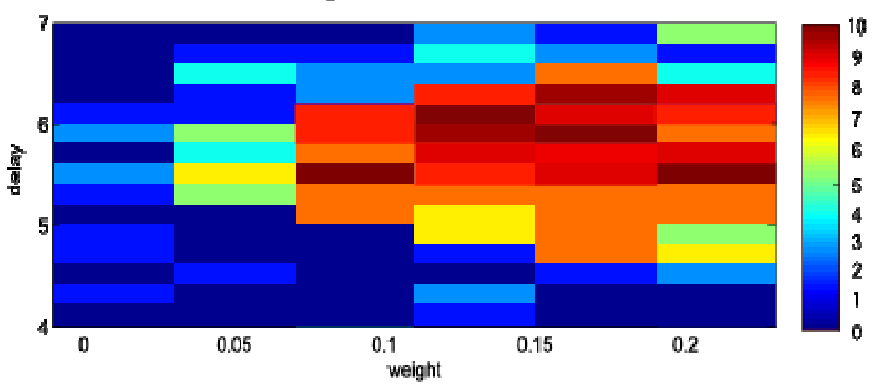

a)

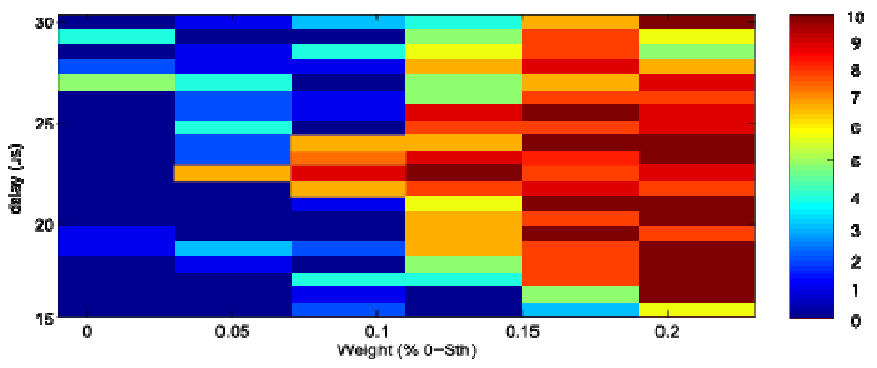

b)

Fig.7: SQ obtained from the model, varying the free parameters in the neighbourhood of the ideal set of values: a) Matlab simulation; b) PSpice simulation . 


\section{CONCLUSIONS}

In this paper we have presented the proof-of-concept for an electronic oscillator based on the neuro-inspired mechanism "dynamical relaying", which relies on a structure composed of three delay coupled oscillating units (as neurons or even neuron populations) able to resonate and self-organise to generate and maintain a given rhythm with great reliability over a considerable parameter range. Although the same structure supports different synchronization regimes, our work has been pointed to one of these, the so-called driven synchrony regime (where the central node oscillates in antiphase with respect to the outer nodes), because it is asintotically stable [17], then suitable for many scenarios. Studies in the field of computational neuroscience have shown that with this kind of motif we can even generate oscillations composed of different frequencies [12], that leave room for the realization of resonant multifrequency oscillators (see [38]), based on the dynamical relaying mechanism. The possibility of using this mechanism for multirhythmic oscillators, together with the intrinsic bidirectionality between the central and the external nodes, suggests an integration into consumer, networking and industrial fields, as for example, security access algorithms for bidirectional data transmissions [39],[40].

\section{ACKNOWLEDGMENTS}

GS acknowledges financial support by the Spanish Ministry of Economy and Competitiveness (PTA-201510395-I)

\section{REFERENCES}

[1] E. A. Vittoz, M. G. R. Degrauwe and S. Bitz, "High-performance crystal oscillator circuits: theory and application," in IEEE Journal of Solid-State Circuits, vol. 23, no. 3, pp. 774-783, June 1988.

[2] A. S. Sedra, "The current conveyor: history and progress," IEEE International Symposium on Circuits and Systems,, Portland, OR, 1989, pp. 1567-1571 vol.3.

[3] W. Pang, R. C. Ruby, R. Parker, P. W. Fisher, M. A. Unkrich and J. D. Larson, "A Temperature-Stable Film Bulk Acoustic Wave Oscillator," in IEEE Electron Device Letters, vol. 29, no. 4, pp. 315318, April 2008.

[4] M.H. Perrott, J.C. Salvia, F.S.Lee., A.Partridge et al. "A Temperature-to-Digital Converter for a MEMS-Based Programmable Oscillator With Frequency Stability and Integrated Jitter," in IEEE Journal of Solid-State Circuits, vol. 48, no. 1, pp. 276-291, Jan. 2013.

[5] M. Zhu and J. L. Hall, "Short and long term stability of optical oscillators," Proceedings of the 1992 IEEE Frequency Control Symposium, Hershey, PA, USA, 1992, pp. 44-55. doi: 10.1109/FREQ.1992.270036.

[6] A.Simonetta, M.C. Paoletti, "Designing Digital Circuits in MultiValued Logic," in International Journal on Advanced Science, Engineering and Information Technology, vol.8, n.4, 2018.

[7] F.Alibart, S.Pleutin, O.Bichler, C.Gamrat, T.Serrano-Gotarredona, B.Linares-Barranco, D.Vuillaume, "A memristive nanoparticle/organic hybrid synapstor for neuroinspired computing", in Adv.Funct.Matter, vol.22, 2012

[8] Y. Hendrawan and D.F.Al Riza, "Machine Vision Optimization using Nature-Inspired Algorithms to Model Sunagoke Moss Water Status," in International Journal on Advanced Science, Engineering and Information Technology, vol.6, n.1, 2016.

[9] I. Fischer, R. Vicente, J.M. Buldu', M. Peil, C.R. Mirasso, M. C. Torrent, J. Garcia-Ojalvo, "Zero-Lag Long-Range Synchronization via Dynamical Relaying," in Phis Rev Lett, vol.97 n.12. Sept. 2006.

[10] R. Vicente, L.L. Gollo, C.R. Mirasso, I. Fischer, and G. Pipa, "Dynamical relaying can yield zero time lag neuronal synchrony despite long conduction delays," Proceedings of the National Academy of Sciences, vol.115, n.44, 2008.
[11] S. Baillet, "Magnetoencephalography for brain electrophysiology and imaging," in Nature neuroscience 20, pp.327-339 , 2017.

[12] L. Gollo, C. Mirasso, O. Sporns, M. Breakspear, "Mechanisms of Zero-Lag Synchronization in Cortical Motifs," in Plos Computational biology, 2014

[13] X.Zhao F. Liu, J.Wang and T.Li, "Evaluating Influential Nodes in Social Networks by Local Centrality with a Coefficient". ISPRS Int. J. Geo-Inf. 2017.

[14] A. Detti, L. Bracciale, P. Loreti, G. Rossi, N. Blefari Melazzi, "A cluster-based scalable router for information centric networks," in Computer networks, pp.24-32, vol.142, 2018.

[15] A. Detti, M.Orru, R.Paolillo, G.Rossi, P. Loreti, L.Bracciale, N. Blefari Melazzi, "Application to information centric networking to nosql database," in 2017 IEEE International Symposium on Local and Metropolitan Area Networks (LANMAN), 2017.

[16] I. Gomes Da Silva, J.M. Buldu, C.R. Mirasso, J.Garcia Ojalvo, "Synchronization by dynamical relaying in electronic circuit arrays," in Chaos 16, 2006

[17] A.Viriyopase, I.Bojak, M.Zeitler and S. Gielen, "When long-range zero-lag synchronization ios feasible in cortical networks," in Frontiers in Computational neuroscience, p.49, vol.6, 2012.

[18] M. Salerno, G. Susi, and A. Cristini, "Accurate latency characterization for very large asynchronous spiking neural networks," in M. Pellegrini, A. L. N. Fred, J. Filipe, and H. Gamboa, editors, BIOINFORMATICS 2011 - Proceedings of the International Conference on Bioinformatics Models, Methods and Algorithms, pages 116-124. SciTePress, 2011.

[19] G. C. Cardarilli, A. Cristini, L. Di Nunzio, M. Re, M. Salerno, and G. Susi, "Spiking neural networks based on LIF with latency: Simulation and synchronization effects," 2013 Asilomar Conference on Signals, Systems and Computers, pages 1838-1842, Pacific Grove, CA, USA, 2013. IEEE.

[20] A. Cristini, M. Salerno, and G. Susi, "A continuous-time spiking neural network paradigm"in S. Bassis, A. Esposito, and F. C. Morabito, editors, Advances in Neural Networks: Computational and Theoretical Issues, pages 49-60. Springer International Publishing, 2015. ISBN 978-3-319-18163-9. doi: 10.1007/978-3-319-18164-6 6.

[21] G. Susi, A. Cristini, and M. Salerno, "Path multimodality in Feedforward SNN module, using LIF with latency model," in Neural Network World, 26(4): 363-376, 2016

[22] G. Susi, L. Anton Toro, L.Canuet, M.E. Lopez, F. Maestu, C.R. Mirasso, and E.Pereda, "A neuro-inspired system for online learning and recognition of parallel spike trains, based on spike latency, and heterosynaptic STDP”. in Frontiers in Neuroscience, 12:780, 2018. ISSN 1662-453X. doi: 10.3389/fnins.2018.00780.

[23] S.Acciarito, A.Cristini, L.Di Nunzio, G.M.Khanal, G.Susi, "An aVLSI driving circuit for memristor-based STDP." IEEE Prime, Lisbon, 2016

[24] S. Acciarito, G.C. Cardarilli, A. Cristini, L. Di Nunzio, R. Fazzolari, G.M. Khanal, M. Re, and G. Susi, "Hardware design of LIF with latency neuron model with memristive STDP synapses," in Integration, the VLSI Journal, 59: 81-89, 2017. ISSN 0167-9260

[25] G. Buzsaki, \& A. Draguhn, "Neuronal oscillations in cortical networks." Science, 304(5679), 1926-1929, 2004.

[26] G.Susi, J.de Frutos-Lucas, G. Niso, S.M. Ye-Chen, L.Antón Toro, B.N.Chino Vilca, F.Maestú, "Healthy and Pathological Neurocognitive Aging: Spectral and Functional Connectivity Analyses Using Magnetoencephalography." in Oxford Research Encyclopedia of psychology and aging. Oxford University press, 2019.

[27] W.O. A.S. Wan Ismail, M. Hanif, S. B. Mohamed, Noraini Hamzah, Zairi Ismael Rizman, "Human Emotion Detection via Brain Waves Study by Using Electroencephalogram," in International Journal on Advanced Science, Engineering and Information Technology, vol.6, n.6, 2016.

[28] G.M. Khanal, S. Acciarito, G.C. Cardarilli, A. Chakraborty, L.D. Nunzio, R. Fazzolari, A. Cristini, M. Re and G. Susi. "Synaptic behaviour in $\mathrm{ZnO}-\mathrm{rGO}$ composites, thin film memristor," in Electronics letters, vol.53, n.5, 2017.

[29] S.S. Saharuddin, N. Murli, M. Azani Hasibuan, "Classification of Spatio-Temporal fMRI Data in the Spiking Neural Network," in International Journal on Advanced Science, Engineering and Information Technology, vol.8, n.6, 2018.

[30] Yuhandri, S.Madenda, E.Prasetyo Wibowo, Karmilasari, "Pattern Recognition and Classification Using Backpropagation Neural Network Algorithm for Songket Motifs Image Retrieval," in 
International Journal on Advanced Science, Engineering and Information Technology, vol.7, n.6, 2017.

[31] A.A. Amri, A.Ritahani Ismail, A.Ahmad Zarir, "Convolutional Neural Networks and Deep Belief Networks for Analysing Imbalanced Class Issue in Handwritten Dataset," in International Journal on Advanced Science, Engineering and Information Technology, vol.7, n.6, 2017.

[32] T.T. Khuat, M.H. Le, "An Application of Artificial Neural Networks and Fuzzy Logic on the Stock Price Prediction Problem," in JOIV International Journal on informatics visualization, vol.1, n.2, 2017.

[33] G. Lo Sciuto, G. Susi, G. Cammarata, and G. Capizzi. A spiking neural network-based model for anaerobic digestion process. In IEEE 23rd International Symposium on power electronics, electrical drives, automation and motion (SPEEDAM), pages 1838-1842, Anacapri, Italy, 2016. IEEE.

[34] S. Brusca, G. Capizzi, G. Lo Sciuto, and G. Susi. A new design methodology to predict wind farm energy production by means of a spiking neural network based-system. International Journal of Numerical Modelling: Electronic Networks, Devices and Fields, 7 2017.
[35] M.S. Meon, M.A. Anuar, M.H.M.Ramli, W.Kuntjoro and Z. Muhammad, "Frame Optimization using Neural Network," in International Journal on Advanced Science, Engineering and Information Technology, vol.2, n.1, 2012.

[36] T.T. Nakagawa, M.Woolrich, H.Luckhoo, M.Joensson, H.Mohseni, M.L.Kringelbach, V.Jirsa, G.Deco, "How delays matter in an oscillatory whole-brain spiking-neuron network model for MEG alpha-rhythms at rest," in Neuroimage, vol.15, n.87, 2014.

[37] A.Mazzoni, H.Linden, H.Cuntz, A.Lansner, S.Panzeri, G.T.Einevoll, "Computing the Local Field Potential from Integrate and Fire Network Models," in PLOS Computational Biology, 2015.

[38] S.Luck, A.Pikovski, 2011 "Dynamics of multi-frequency oscillator ensembles with resonant coupling" in Phisics letters A, vol. 375 , issues 28-29, 2011

[39] R.Giuliano, F.Mazzenga, A.Neri, A.M.Vegni, "Security Access Protocols in IoT Capillary Networks," IEEE Internet of Things Journal 4 (3), 645-657, 2017.

[40] R.Giuliano, F.Mazzenga, A.Neri, A.M.Vegni, "Security Access Protocols in IoT Capillary Networks with heterogenous non-ip terminals," in 2014 IEEE International Conference on Distributed Computing in Sensor, 2014. 